\title{
1. Introduction to Technology, Innovation and Healthcare
}

\section{BACKGROUND}

The doctor/patient relationship was once a relatively simple one. It developed in a bricks and mortar environment, there was a face to face, often quite paternalistic, interaction in which the doctor shared his knowledge and wisdom, guiding the patient down a treatment path. ${ }^{1}$ The nature of this relationship has evolved and changed. We now see a more empowered patient and a shift towards a relationship that is a more equal partnership. Alongside this shift, the nature of healthcare has changed. Long gone are the days of amputation of broken limbs, or the description of anaesthesia as a 'needless luxury', ${ }^{2}$ and we can adapt the words of Heraclitus and assert that the only constant in medicine is change. The clinical landscape has become more complex and sophisticated, and as technology plays an increasingly significant role in healthcare, there is the prospect of a new imbalance in power. One that could negatively impact on both patients and healthcare professionals if their interests are not adequately protected, as innovation continues to change the possibilities of action for both. It is this growing role of technology, and its impact on healthcare, that provide the backdrop for this book.

Technology is a broad term, and in the context of healthcare it can be applied to a seemingly endless series of tools, processes and devices. There are technological innovations available in nearly all clinical interactions including (but not limited to): surgical interventions, patient monitoring, health screening and diagnosis, research and the collection and interpretation of patient data. In truth, health technology is as broad as healthcare itself and it is this diversity, combined with the rapid advancement of health technology, that is presenting a challenge to its regulation in the context of healthcare. The most common

1 This is of course a stereotypical portrait of the doctor, and deliberately gendered given the reality that originally the doctor was invariably a male and the relationship with the patient was indeed a paternalistic one.

2 A. Gawande (2012), 'Two hundred years of surgery', New England Journal of Medicine, 366(18), 1716-1723. 
narrative is that technology is advancing at such a rate that 'antiquated legal institutions [are being outstripped] in the blinding rush of progress' . ${ }^{3}$ It is not just the pace of change that is seen to represent a challenge to the law, but the breadth of change may also be seen to challenge the adequacy of any regulatory response. In seeking to write a book about regulation and its relationship to technology, innovation and healthcare, we could be accused of presenting as unified a series of issues connected only tangentially by association with a particular subject matter. Judge Easterbrook once famously critiqued examination of property law in cyberlaw, decrying it as akin to the study of 'the law of the horse'. ${ }^{4}$ He suggested it is far better to properly understand the general law and then apply it to a particular thing, than to collect together all the applications of law in relation to that one thing and then seek unifying themes. As explained by Easterbrook:

... the best way to learn the law applicable to specialized endeavors is to study general rules. Lots of cases deal with sales of horses; others deal with people kicked by horses; still more deal with the licensing and racing of horses, or with the care veterinarians give to horses, or with prizes at horse shows. Any effort to collect these strands into a course on 'The Law of the Horse' is doomed to be shallow and to miss unifying principles. ${ }^{5}$

Indeed, what we are attempting might be seen even less favourably than an attempt to describe the 'law of the horse'. At least the concept of a horse is relatively uncontested. As we shall see throughout this book, we are constantly encountering concepts, including the concepts of technology, healthcare and innovation, which themselves have blurred boundaries and contested definitions. The question then is, can we truly discuss the general relationship between healthcare, technology and the law or does this represent a doomed endeavour?

It is clear that in our view, it is not only possible - but appropriate - to consider the evolving relationship between the law and technology, innovation and healthcare. We do not seek to describe the 'law of technology and innovation in healthcare'. Instead, we reflect on the changes that we see as innovations increasingly embedding technology in healthcare and use that as motivation

3 Joshua A.T. Fairfield (2021), Runaway Technology, Cambridge: Cambridge University Press, Kindle edition, p. 7.

4 For a detailed discussion of the 'law of the horse' see Lawrence Lessig (1999), 'The law of the horse: what cyber law might teach', Harvard Law Review, 113(2), 501-549, and Frank H. Easterbrook (1996), 'Cyberspace and the law of the horse', University of Chicago Legal Forum, 1, Article 7, 207.

5 Frank H. Easterbrook (1996), 'Cyberspace and the law of the horse', University of Chicago Legal Forum, 1, Article 7, 207. 
to consider the adequacy of current regulation. We embarked on the writing of this book with two certainties: the first being that technology in healthcare presents a regulatory challenge and the second, that there is a regulatory gap that must be filled. In essence, both of these certainties remain (although our view is now far more nuanced), and along the way we have gathered a number of uncertainties and considered a diverse range of challenges that are found in this space.

The environment we are considering is a dynamic one and the role of technology in the provision of healthcare is changing at a significant rate; it is difficult for any single group of professionals to keep pace with this development. Additionally, it is virtually impossible for any single regulatory regime to address the multitude of issues that arise. As we noted above, innovation and technology impact on every aspect of the provision of healthcare and attempts to respond with individual legal reform are akin to putting out small spot fires in the face of a major inferno. However, it is not just healthcare that is changing; we live in a world where the accepted position is that change is good, innovation is automatically perceived as better, and each new day brings new technology. In this environment, mortality is hard to grasp, and the idea of an incurable disease is incomprehensible to most. There is a perception that the brave new world of technological advancement is a better place where disease will be banished and human error overcome. Whilst technological advancement has the potential to improve healthcare, achieve longer life and reduce the impact of human error, it brings with it a new set of legal and ethical concerns that warrant careful consideration. If we rush headlong into this 'brave new world', we run the risk of overlooking some significant issues. In this book we seek to engage with some of the issues arising in different areas of healthcare and the law and explore the specific challenges that are presented.

\section{THE STRUCTURE OF THE BOOK}

Our discussions in this book move from the general (a broad overview of the nature of innovation and regulation) through to more focussed discussions addressing topics including patient consent, the role of device representatives, privacy, artificial intelligence and big data. In exploring these topics, we covered a lot of conceptual and theoretical ground; yet as we worked through the diverse challenges represented under each topic, we found that two themes emerged: disruption and trust. We have shaped our definition of innovation around the concept of disruption and this theme then threads its way through all of our discussions. The second overarching theme is that of trust, which spans a broad range of issues including expectations (of both doctor and patient), integrity, competence, quality control and experience. 
We have divided the book into three parts, each presenting a slightly different perspective. This first part is all about setting the scene, with this chapter providing an overview of the book and our themes and then Chapter 2 providing essential context for the rest of the book and establishing our definition of innovation. Part II focusses on innovation and the patient, exploring the question of patient safety through an effective regulatory framework and the complexities of appropriate patient consent to innovative treatment. This then leads into the final chapter in Part II, which challenges the role of medical device representatives in healthcare. Part III then moves on to consider the broader concept of innovation as the future of healthcare. We begin this part with a careful critique of the regulatory approach to the use of artificial intelligence in healthcare; this is a general discussion introducing some broad ideas that are explored in more detail in the final two chapters, which have a narrower focus, first on questions around protection of privacy, and finally, on the related area of algorithms, trust and the use of big data in healthcare decisions.

\section{TRUST AND DISRUPTION}

Before engaging more fully with the different chapters of the book, we invite you now to consider the twin themes of trust and disruption and how they are woven through each chapter. Chapter 2 is all about disruption through innovation and sees the introduction of many of the recurring ideas that are woven through the following chapters. This chapter presents a foundation that we build on as we progress through considerations of different examples of innovation in healthcare. It is here that we first raise the question regarding the nature of regulation of innovation, which is then expanded on in Chapter 3. We also begin with the presumption that constant change and improvement are crucial in the context of healthcare. However, we emphasise from the outset that innovation must take place in a transparent, careful and responsible way, and much of the book deals with how this can occur.

In this chapter we also introduce the importance of considering the provision of healthcare as a multilayered, complex interplay of different participants. To simply focus on any individual group is to fail to address the true complexities present at the intersection of healthcare, technology and the law. We must consider all participants - from the broadest group represented by the healthcare system, through the developers of technology, healthcare providers, those who make and sell devices, the individual patients, broader society and even humanity itself. Here we see the significance of relationships and thus of trust.

A further important point that we make in Chapter 2 is that whilst innovation can bring some harms, it also brings enormous potential for benefits, many of which are highlighted throughout the broader discussions presented. This chapter also explores the different 'logics' that impact on the introduction of 
new technologies; these are presented as drivers of change, and the important idea that they are much broader than individual patient need is introduced. ${ }^{6}$

Chapter 3 builds on the regulatory challenges introduced in Chapter 2 and outlines the complex task of developing regulation in the context of the disruption created by the introduction of technology into healthcare and the ongoing innovation in this space. Appropriate regulation plays a key role in establishing trust between society, healthcare providers and individual patients. The traditional focus of healthcare regulation has been on the protection of patient safety; this chapter engages with this and challenges this focus, suggesting instead that a risk/benefit analysis that strengthens the values of trust and explainability is now emerging.

Chapter 3 also provides an important framework for the discussions to follow as it engages with the nature of innovation and emphasises the need for effective, transparent and flexible regulation if the introduction of technology into healthcare is to be safe. Appropriate regulation will not only support safety in healthcare, but it will serve to engender trust and encourage innovation. This will not occur if an inflexible and narrow regulatory framework is created; in the chapter we argue that effective (and thus appropriate) regulation is context-specific. The challenges presented by technology in healthcare require a broad ranging, multilayered approach that extends from the licensing of devices or practices through to the embracing of the commercial reality which involves consumer protection law. This is not inconsistent with traditional healthcare regulation, as that is also protective in nature. Whilst the chapter provides a case study of regulation and approaches to regulation of healthcare in Australia, the discussion is of global relevance as it identifies different modes of regulation, from professional codes, through statutory instruments, to the newest player in this sphere, consumer protection law.

In Chapter 4 we pick up on some of the challenges highlighted in Chapter 2, in which we considered the complexities around innovation in the healthcare space. Chapter 4 engages with some of the issues that arise here from the viewpoint of the patient who, in the face of truly innovative healthcare, is asked to agree to risks - and potentially benefits - that are unknown. We also pick up on the therapeutic misconception that was introduced in Chapter 2, that is, the belief that because something is new, it offers a heightened level of hope. Here we consider a very real challenge to the doctor/patient relationship of trust, because when that hope is not met, unsurprisingly, the clinical relationship is

6 The idea of 'logics of change' is introduced in C. Berggren and S.T. Karabag (2019), 'Scientific misconduct at an elite medical institute: the role of competing institutional logics and fragmented control', Research Policy, 48(2), 428-443, where they are presented as including patient care, institutional need, financial benefit/cost and government imperatives. These are explored in more detail in Chapter 2. 
harmed and trust, not only in the individual doctor but also in the provision of healthcare in general, declines. The idea that 'innovative' or new treatment can provide hope where there was none has given rise to the 'right to try' movement which has gained significant traction in the USA and was at the centre of much debate in the UK. This model of patient-driven access to unproven treatment is explored in the chapter as it provides a different perspective in the consent discussion and considers the shift from consent to demand for treatment.

A final theme introduced in Chapter 4 is the concept of 'trespass by technology', when we ask the question: does the introduction of an alternate means of providing treatment serve to act as a trespass as it changes the character of the treatment and is akin to performing a different treatment altogether? Here the doctor/patient relationship is disrupted through the introduction of novel forms of treatment and patient trust is undermined because the patient is not informed about the change to the treatment plan.

In Chapter 5 we move on to consider a specific situation in which the introduction of technology into the clinical relationship has evolved in such a way as to, potentially, disempower the patient and, once again, disrupt the clinical relationship and erode trust. The introduction of highly technical devices in the care of patients presents a challenge to clinicians; these devices are often sophisticated in design and are outside general clinical experience and expertise. To meet this challenge, it is becoming increasingly common practice for industry representatives to be present in hospitals, providing guidance and advice to treating clinicians. Industry representatives provide support for the use of devices supplied by the companies for whom they work and are often driven by a commercial imperative that is a direct contrast to the clinical imperative of the treatment team. Activities performed by representatives include being present in operating theatres to provide technical guidance to surgeons and nursing staff; to 'troubleshoot' equipment problems on the wards; and to provide in-house training to staff using new devices and technologies. The introduction of these industry representatives into the clinical environment potentially disrupts the treatment path and clinical relationship between the doctor and patient and, as explored through some case studies, serves to undermine not only individual but also public trust in clinicians, device representatives and the broader healthcare system.

The third part of the book looks to innovation as being the future of healthcare as we anticipate non-human actors having an increasingly significant role in the development and delivery of healthcare. We open Part III by returning to the consideration of a regulatory gap. We consider the situation where the use of technology in healthcare is common and address questions of responsibility and liability when things go wrong, particularly when it is not clear, due to the nature of the technology, what has caused an error or adverse outcome. We 
return here to the idea of disruption but approach it from the perspective of responsibility. The focus in this chapter is on the use of artificial intelligence (AI) in healthcare and one of the first stumbling blocks we identify is the difficulty of adequately defining AI and delimiting its use. The main substance of the chapter is a consideration of the challenge of attributing responsibility when the clinical outcome is suboptimal. We consider the complex issue of how to define relevant harm and argue for a broad view. If any regulatory gap is to be adequately addressed, then we must consider the nature of harms which may extend beyond straightforward physical harm. The harms we identify include potential reputational and professional harms to healthcare professionals, changing clinical interactions and, potentially, creating a less personal clinical experience for patients.

This chapter engages once again with models of regulation and considers not only who may be responsible but also - having decoupled the question of responsibility from that of legal liability - who, and what, should be regulated. Health regulation is typically targeted at the clinical interface, but when we are exploring the role of $\mathrm{AI}$ in health there is an argument for taking a broader view when considering who may be the most appropriate target of regulation. Responsibility can extend from the original code writer through to the clinician (or technician) who provides the treatment or perhaps even to the AI itself. We work through some of the models of responsibility that are discussed in the literature and present this as a 'continuum of responsibility', ranging from the instrumentalist view (which would assign responsibility to those who devise, use and benefit from the technology), through the many hands perspective (which would assign responsibility jointly to those who collectively contribute to the development of an AI system), to computer/AI responsibility (which would assign responsibility to the AI system itself as an independently functioning entity). While there are points of persuasion in relation to each, we conclude the discussion with our view that the most persuasive model is a development of existing principles of contribution to harm. While it may be difficult to tease out individual contributions, it is unlikely to be impossible (particularly if systems have been designed to support it) and legal responsibility should normally be assigned according to capability to take the action necessary to avoid unjustified harm.

This then leads us into the final two chapters which, by design, apply a broader, more theoretical lens to some of the issues raised by increasing reliance on non-human actors in healthcare and healthcare innovation. In Chapter 7 we consider the question of what privacy means in an era of big data. With the temptation to harness the power of big data analytics to address some of the profound challenges facing healthcare systems around the world today proving irresistible, we ask: what are the implications for privacy and the adequacy of current legal protection? To answer this question, we begin by considering the 
concept of privacy and, with a focus on informational privacy, consider it from the perspective of state of separateness, social norm, and legal right. This highlights the value-laden and purposive dimension of privacy protection, with the identification of material states of separation reliant upon value judgements, and social norms associated with a desire that social actors be accountable for external costs and benefits of their behaviours. Legal protections for data privacy need to be capable of adapting as the costs and benefits of behaviour change across time, particularly where other norms might be inchoate or ineffective and identification of material states of separateness become outdated.

Our principal argument in Chapter 7 is that, in order to appreciate the regulatory gap that exists in relation to the protection of privacy in an era of big data, the law must step up to regulate use and reuse of data in the context of healthcare innovation, both where data relates to individuals as members of groups, and where data relates to groups and not only to identified or identifiable individuals. The approach that seems most suited is one that can continue to protect reasonable expectations of privacy after data has been disclosed by an individual or group and can regulate 'downstream' uses of data even if it has been aggregated or anonymised or other data has been derived from it. In this regard, there are limitations to the traditional approaches to protecting privacy as an aspect of liberty or human dignity in so far as the former can be associated with what has been described as the secrecy paradigm - so that which is no longer secret can no longer be private - and both have been associated with atomistic individualistic conceptions of privacy. Perhaps this association is most readily apparent in the law's definition of 'personal data', which delimits the scope of protection. If data does not relate to an identified or identifiable individual, then it is not protected. We argue here that, consistent with our position elsewhere in the book, rather than radical invention of a new legal framework, the most appropriate response may be through the evolutionary development and application of existing legal principles. In this case, we argue that the traditional law of confidence might be pressed to do some valuable work in protecting reasonable expectations of privacy even where data does not relate to identifiable individuals. This is essential, we suggest, if the law is to protect our collective interests in privacy into the future and to properly regulate and enable access to health data for the purposes of healthcare innovation.

Enabling appropriate use of data will be just as important to the future of healthcare innovation as disabling inappropriate use. In Chapter 8, we consider what will be required of health data governance into the future if it is to protect and promote public trust and confidence in appropriate uses of data. In 'Algorithms, trust and the use of big data in healthcare decisions', we move beyond questions concerning the allocation of responsibility for harm and privacy intrusion, to consider what is required of AI systems operating in the 
context of healthcare if they are to be trustworthy from the patient perspective. Having noted the rise of big data in healthcare, we recognise the distinction drawn in the literature between the use of AI systems to do things that healthcare professionals are doing now and their use to do things that humans have not been able to do before. In so far as trustworthiness requires beneficence, there is an open question with regard to the extent of the responsibility that is to be placed on AI systems (and, in particular, commercial organisations responsible for their development, deployment and use) to do more than avoid harm and to do good: to act positively in the interests of the patients who rely on them. Where AI systems are doing things that healthcare professionals have previously done, or could otherwise do, the well-placed trust of a patient is going to require that such systems operate for them at least as well as a health professional would. Otherwise, whatever other standard such systems might meet, this failure will undermine a system's trustworthiness from a patient perspective. Where systems are breaking new ground and offering healthcare benefits that are otherwise unobtainable from human actors, then a novel calculation of trade-offs (e.g., against privacy) will be required before acceptability of a system can be determined. However, our argument is that we must not lose sight of the basic requirement that a trustworthy system requires not only technical competence, and integrity (by which we mean congruence with other values), but also beneficence. We must always consider who benefits from the introduction of systems and to require that those affected by them do themselves have reason to accept their introduction. This requirement is a bare minimum for trustworthiness and for such systems to operate in the public interest. It should not be avoidable by any reliance upon individual consent or the anonymisation of data. We must look forward to a system of health data governance that shoulders the responsibility for not only protecting people from unfair uses of data, but enables fair use and positively promotes healthcare innovation in the public interest.

Disruption and trust thus thread their way throughout all of the discussions in this book and emerge as dominant themes. Perhaps a simple way to summarise the core question considered in this book is to ask: can regulation serve to either protect or restore trust in the face of the disruption caused by the introduction of technology into healthcare? We have engaged with this question in a number of different contexts throughout the book and whilst there is no single or clear answer there are a number of principles that can help guide us through this disruption. We did not set out to identify a regulatory framework or to provide a single guiding principle. Our aim was to engage in relevant dialogue and contribute to the ongoing debates about the intersection of technology, healthcare and the law. We invite you to read through this book as it interests you; there is no need to read all of the chapters, or to read them 
in a specific order. If we add to the conversation and engage your interest, then we have met our primary aim. 